\title{
Floating Sheath Potentials in Non-Maxwellian Plasmas
}

\author{
M. J. KUSHNER
}

\begin{abstract}
The floating sheath potential in a plasma having a Maxwellian electron distribution function is $e \phi_{s}=-k T_{e} \ln (a / b) / 2$ where $T_{e}$ is the electron temperature, $a$ is the ratio of electron temperature to ion temperature, and $b$ is the ratio of electron mass to ion mass. This expression is derived by equating the flux of electrons and ions to a surface in the plasma. Only electrons initially having an energy greater than $-e \phi_{S}$ flow to the surface. These electrons are in the tail of the distribution, a region that differs significantly from a Maxwellian in many plasmas. An analysis is performed where the sheath potential is solved for using a two-temperature model for the electron distribution function. The two-temperature model accurately describes the distortion from a Maxwellian in the tail of the distribution function. The magnitude of the sheath potential calculated with the two-temperature distribution is significantly smaller than that obtained using a Maxwellian distribution, a result of the reduction in the relative abundance of energetic electrons in the tail of the distribution.
\end{abstract}

W HEN IMMERSED in a plasma, an insulator or isolated conductor will acquire a negative charge [1]. Acquisition of the charge is caused by the disparity in mobility between electrons and ions. Electrons, having a much higher mobility and smaller mass than ions, have a correspondingly higher thermal or random current in the plasma. This condition is exacerbated when the electron temperature is higher than the ion temperature. Due to the electrons having a larger random current, the surface immersed in the plasma initially collects more electrons than ions, thereby collecting an excess of negative charge. Since in the steady state the surface cannot continue to collect net charge, a negative potential develops at the surface to retard the electron flux. The electron flux is lowered to a value equal to that of the ion flux. This retarding electrical potential is called the sheath potential, and the nonneutral region adjacent to the surface is called the sheath. The sheath is typically a few to tens of Debye lengths thick. The magnitude of the sheath potential can be solved for by assuming the electron and ion distribution functions are Maxwellians, the sheath is collisionless, and by equating the values of the electron and ion fluxes entering the sheath [1]. Doing so, the sheath potential $\phi_{s}$ is given by

$$
e \phi_{s}=-k T_{e} \ln \left(T_{e} M /\left(T_{I} m\right)\right) / 2 .
$$

In (1), $T_{e}$ is the electron temperature, $T_{I}$ is the ion temperature, $m$ is the electron mass, and $M$ is the ion mass. The sheath potential is typically a few to ten times the electron temperature.

As a consequence of the negative potential acquired by the surface, only electrons entering the sheath with kinetic energy

Manuscript received May 29, 1984; September 18, 1984.

The author is with Mathematical Sciences Northwest, Inc., (Spectra Technology, Inc.), Bellevue, WA 98004. greater than the sheath potential can be collected by the surface. These particles have energies many times the average electron energy and reside in the tail of the electron distribution. It is well known, though, that electron distribution functions differ from a Maxwellian most markedly at higher energies, especially for energies greater than the first inelastic threshold [2]. Typically, the electron distribution is depleted of electrons with energy greater than this value. Electrons energetic enough to excite the gas atoms or molecules, and do so, lose in energy a value at least equal to the threshold value for excitation. The electron typically rejoins the distribution at an energy below the threshold value. The electron distribution is therefore "cut off" at an energy given by the first excitation threshold. Since it is the more energetic electrons that are collected by a surface immersed in a plasma, assuming a Maxwellian electron distribution overestimates the electron flux to the surface, and hence overestimates the sheath potential.

A complete description of the sheath region near a surface in a plasma requires one to simultaneously solve Poisson's and Boltzmann's equations for the electrons and ions in a region many Debye lengths thick adjacent to the surface. The effect of interest, that of the change in sheath properties resulting from a non-Maxwellian electron distribution, can be studied in some detail with a simpler analysis to be discussed here. In this analysis, a solution for the electron distribution function, called a two-temperature model, is used [3]-[5]. In this solution, the electron distribution function is assumed to be the continuation of two Maxwellian distributions with separate electron temperatures $T_{1}$ and $T_{2}$. For electron energies less than a cutoff energy $E_{c}$, typically equal to the first excitation threshold, the distribution is a Maxwellian with temperature $T_{1}$. For electron energies greater than the cutoff energy, the distribution is a Maxwellian with temperature $T_{2}$. To simulate the cutoff behavior of the true distribution, we must have $T_{2}<T_{1}$. The two electron groups are referred to as the bulk (first group) electrons and the tail (second group) electrons. The normalization constants for the two segments of the distribution function are chosen such that the distribution is continuous at $E_{c}$ and that the integral of the distribution function over all energies is unity. That is

$$
\begin{aligned}
& \alpha_{1} f\left(T_{1}, E_{c}\right)=\alpha_{2} f\left(T_{2}, E_{c}\right) \\
& \int_{0}^{E_{c}} \alpha_{1} f\left(T_{1}, \epsilon\right)\left(\frac{2 \epsilon}{m}\right)^{1 / 2} d \epsilon \\
& \quad+\int_{E_{c}}^{\infty} \alpha_{2} f\left(T_{2}, \epsilon\right)\left(\frac{2 \epsilon}{m}\right)^{1 / 2} d \epsilon=1
\end{aligned}
$$


where $f(T, \epsilon)=\exp (-\epsilon / k T)$. The normalization constants are

$$
\begin{aligned}
\alpha_{1}^{-1}= & \left(\left(E_{c} / \chi\right)^{3} \frac{2}{m}\right)^{1 / 2} \\
& \cdot\left[\frac{\sqrt{\pi}}{2} \operatorname{erf}\left(\chi^{1 / 2}\right)-e^{-\chi} \chi^{1 / 2}\right. \\
+ & e^{-\chi(1-\beta)} \beta^{3 / 2}\left[\frac{\sqrt{\pi}}{2}\left(1-\operatorname{erf}\left((\chi \beta)^{1 / 2}\right)\right)\right. \\
& \left.\left.+e^{-\chi \beta}(\chi \beta)^{1 / 2}\right]\right] \\
\alpha_{2} & =\alpha_{1} e^{-\chi(1-\beta)}
\end{aligned}
$$

where $\chi=E_{c} / k T_{1}$, and $\beta=T_{1} / T_{2}$. If $T_{1}=T_{2}$, the distribution is a Maxwellian.

To solve for the sheath properties, we assume the sheath is collisionless for both electrons and ions. The electron and ion density in the plasma at the edge of the sheath is $N_{0}$. The ion velocity at that point is $V_{0}$. By flux and energy conservation, we can solve for the ion density $N(x)$ in the sheath:

$$
\begin{aligned}
N_{0} V_{0} & =N(x) V(x) \\
\frac{1}{2} M V(x)^{2} & =\frac{1}{2} M V_{0}^{2}-e \phi(x) \\
N(x) & =N_{0} /\left(1-2 e \phi(x) / M V_{0}^{2}\right)^{1 / 2} .
\end{aligned}
$$

In (6), $\phi(x)$ is the electric potential. The electron density at a point in the sheath with potential $\phi(x)$ is

$$
n(x)=N_{0} \int_{-e \phi(x)}^{\infty} F(\epsilon) \epsilon^{1 / 2} d \epsilon
$$

where $F(\epsilon)$ is the electron distribution function in the plasma at the edge of the sheath. Equation (7) is simply a statement that only electrons at the edge of the sheath initially having an energy greater than $-e \phi(x)$ will be energetic enough to climb the potential hill at location $x$. For (7) to be valid, we must assume that the absolute magnitude of the potential is a monotonically increasing function of distance between the edge of the sheath and the surface. For our purposes, $F(\epsilon)$ is a twotemperature Maxwellian distribution. The electron density in the sheath is therefore

$$
n(x)=\left\{\begin{array}{rr}
N_{0}\left[\int_{-e \phi(x)}^{E_{c}} \alpha_{1} f\left(T_{1}, \epsilon\right)\left(\frac{2 \epsilon}{m}\right)^{1 / 2} d \epsilon\right. \\
\left.\quad+\int_{E_{c}}^{\infty} \alpha_{2} f\left(T_{2}, \epsilon\right)\left(\frac{2 \epsilon}{m}\right)^{1 / 2} d \epsilon\right], & -e \phi(x)<E_{c} \\
N_{0} \int_{-e \phi(x)}^{\infty} \alpha_{2} f\left(T_{2}, \epsilon\right)\left(\frac{2 \epsilon}{m}\right)^{1 / 2} d \epsilon, & -e \phi(x) \geqslant E_{c} .
\end{array}\right.
$$

With these expressions for electron and ion density, the sheath potential $\phi(x)$ is obtained by directly solving Poisson's equation:

$$
\frac{\partial^{2} \phi(x)}{\partial x^{2}}=-\frac{e N_{0}}{\epsilon_{0}}\left(\frac{1}{\left(1-2 e \phi(x) / M V_{0}^{2}\right)^{1 / 2}}-n(x) / N_{0}\right) .
$$

Equation (9) can be solved by direct integration beginning at the edge of the sheath. The initial conditions are $\phi=0$ and $\partial \phi / \partial x=\delta$, where $\delta$ is a small negative value. Integration continues until $\phi(x)=\phi_{s}$. The distance $x$ at which this occurs is then the sheath thickness. The sheath potential $\phi_{s}$ is obtained by equating the electron and ion fluxes to the surface:

$$
N_{0} V_{0}=\left\{\begin{array}{cc}
N_{0}\left[\int_{-e \phi(x)}^{E_{c}} \alpha_{1} f\left(T_{1}, \epsilon\right)\left(\frac{2 \epsilon}{m}\right) d \epsilon\right. & \\
\left.\quad+\int_{E_{c}}^{\infty} \alpha_{2} f\left(T_{2}, \epsilon\right)\left(\frac{\epsilon}{2 m}\right) d \epsilon\right], & -e \phi_{s}<E_{c} \\
N_{0} \int_{E_{c}}^{\infty} \alpha_{2} f\left(T_{2}, \epsilon\right)\left(\frac{2 \epsilon}{m}\right) d \epsilon, & -e \phi_{s} \geqslant E_{c} .
\end{array}\right.
$$

To generalize the analysis, it is convenient to rewrite (9) in normalized units:

$$
\frac{\partial^{2} \psi}{\partial \eta^{2}}=-\left(1 /(1-\psi \delta / \theta)^{1 / 2}-n(\eta) / N_{0}\right)
$$

where $\psi=e \phi / k T_{1}, \delta=m / M, \theta=T_{I} / T_{1}$, and $\eta^{2}=x^{2} e^{2} N_{0} / k T_{1} \epsilon_{0}$. $\psi$ is the electric potential divided by the first group electron temperature, $\delta$ is the ratio of electron to ion mass, and $\theta$ is the ratio of ion temperature to first group electron temperature. $\eta$ is the spatial coordinate in units of the first electron group Debye length $\lambda_{1}=\left(\epsilon_{0} k T_{1} / N_{0} e^{2}\right)^{1 / 2}$. Similarly, the electron density $n(x)$ can be written in dimensionless form as a function of $\psi, \chi=E_{c} / k T_{1}$ and $\beta=T_{2} / T_{1}$.

The electric potential, electron, and ion densities in the sheath region of a two-temperature plasma, computed in the manner described above, are plotted in Fig. 1. The ratio of electron to ion mass is $1 \times 10^{-5}$, and the ratio of ion temperature to electron temperature is 0.2 . The cutoff energy for the two-temperature distribution is $E_{c}=k T_{1}$. The curves are for different values of the ratio of second group electron temperature to first group electron temperature. The smaller this ratio, the more non-Maxwellian the distribution appears, and the more severely the electron distribution is depleted of electrons with energy greater than $E_{c}$. As $T_{2} / T_{1}$ decreases, both the sheath potential and sheath thickness decrease. The decrease in sheath potential is in part a result of a decrease in the average electron energy but is more a reflection of the necessity of the ion flux to be balanced by more numerous, but less energetic, electrons instead of fewer electrons of higher velocity, now absent from the tail of the distribution. Because the sheath potential is lower with smaller $T_{2} / T_{1}$, the ion energy, and hence velocity, at the surface are smaller. Since the ion flux is conserved, the smaller ion velocity translates to a higher ion density at the surface.

The degree to which the sheath potential is lowered, and sheath thickness reduced, is a function not only of $T_{2} / T_{1}$ but also of the ratio $E_{c} / k T_{1}$, the cutoff energy divided by the first group electron temperature. The sheath potential as a function of these two parameters is plotted in Fig. 2. For a given value of $T_{2} / T_{1}$, the electron distribution is more severely 
(a)

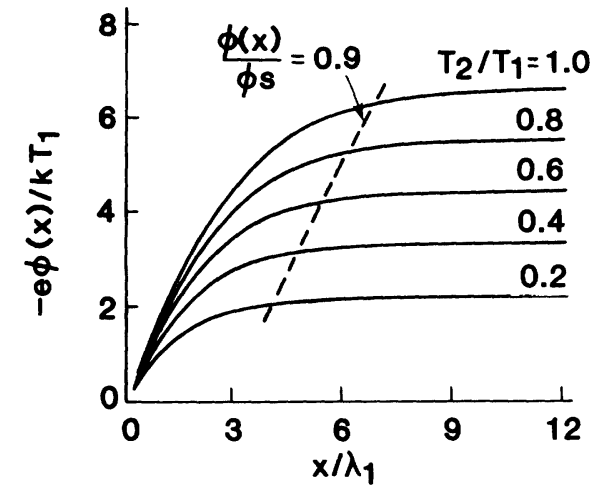

(b)

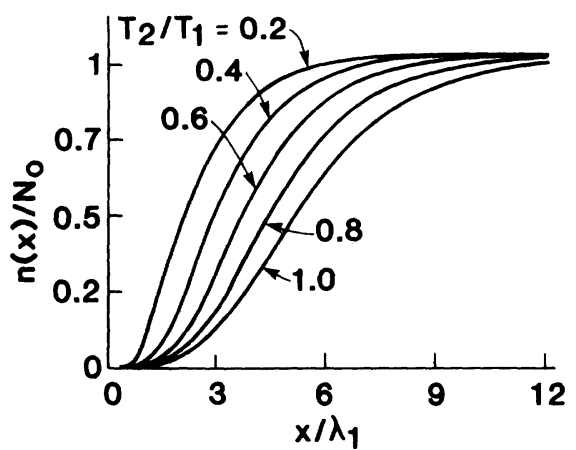

(c)

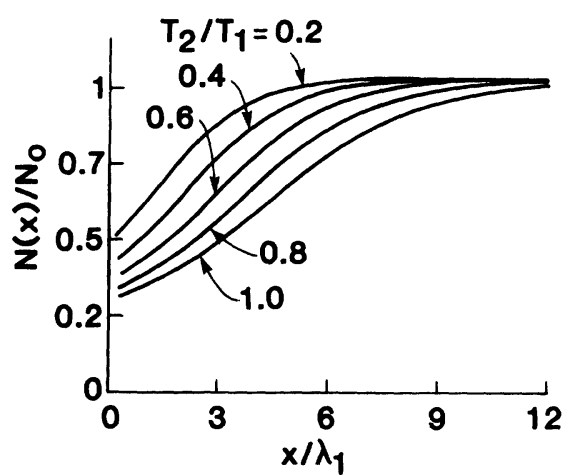

Fig. 1. Computed sheath properties using the two-temperature model. a) Plasma potential measured with respect to the sheath potential (in units of $\left.k T_{1} / e\right)$ as a function of $x / \lambda_{1} .\left(T_{I} / T_{1}=0.2, m / M=1 \times 10^{-5}\right.$ $\left.E_{c} / k T_{1}=1.0\right)$. The dashed line is the locus of points where $\phi(x) / \phi_{s}=$ 0.9 , illustrating the decrease in sheath potential as $T_{2} / T_{1}$ decreases. b) Electron density $\left(n(x) / N_{0}\right)$ and c) ion density $\left(N(x) / N_{0}\right)$ for the same conditions.

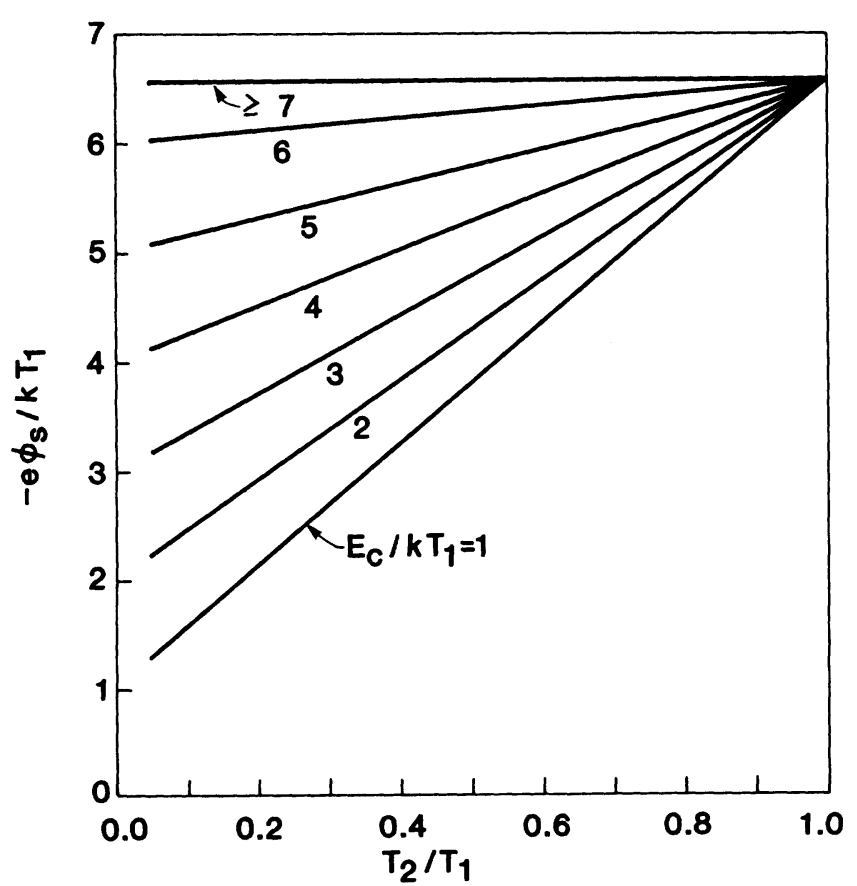

Fig. 2. Sheath potential (in units of $k T_{1} / e$ ) as a function of $T_{2} / T_{1}$ for different values of $E_{c} / k T_{1}$. The sheath potential for a Maxwellian distribution corresponds to $E_{c} / k T_{1} \gg 1$ and $T_{2} / T_{1} \approx 1$.

depleted of high energy electrons for lower values of $E_{c} / k T_{1}$. The sheath potential therefore decreases with decreasing $E_{c} / k T_{1}$. For $T_{2} / T_{1} \approx 1$ and $E_{c} \gg k T_{1}$, the two-temperature distribution is nearly a Maxwellian and the sheath potential is as given by (1).
To obtain the parameters $T_{2} / T_{1}$ and $E_{c} / k T_{1}$ to correct the floating sheath potential for non-Maxwellian effects, one must have some knowledge of the electron distribution function. To obtain these parameters directly, a two-temperature analysis for the electron distribution function must be performed. $T_{2} / T_{1}$ and $E_{c} / k T_{1}$ can also be obtained by other methods; from experimentally measuring the distribution function using electric probes or from numeric solutions of Boltzmann's equation. When extracting $T_{2} / T_{1}$ and $E_{c} / k T_{1}$ from a plot of the electron distribution function, one plots the log of the function versus electron energy. The slope of the plot at a given energy defines the local electron temperature (i.e., $T_{1}$ or $T_{2}$ ). An abrupt change in the slope of the plot defines the cutoff energy $E_{c}$. This method for obtaining $T_{2} / T_{1}$ and $E_{c} / k T_{1}$ is discussed in detail in [6].

In order to apply the theory described in this paper, the homogenous Boltzmann equation for the electron distribution function in the plasma must be solved to obtain the parameters $T_{2} / T_{1}$ and $E_{c} / k T_{1}$. Given these parameters, one can characterize the sheath from the results of this theory as summarized in Fig. 2. This method is much simpler, though, than solving the inhomogenous Boltzmann equation required for an exact description of the sheath. The calculation necessary to obtain the exact description requires the solution of a system of many simultaneous partial differential equations (functions of the spatial coordinate and electron energy) as well as simultaneous solution of Poisson's equation.

For purposes of illustration, the parameters $T_{2} / T_{1}$ and $E_{c} / k T_{1}$ were obtained from solution of the homogenous Boltzmann 

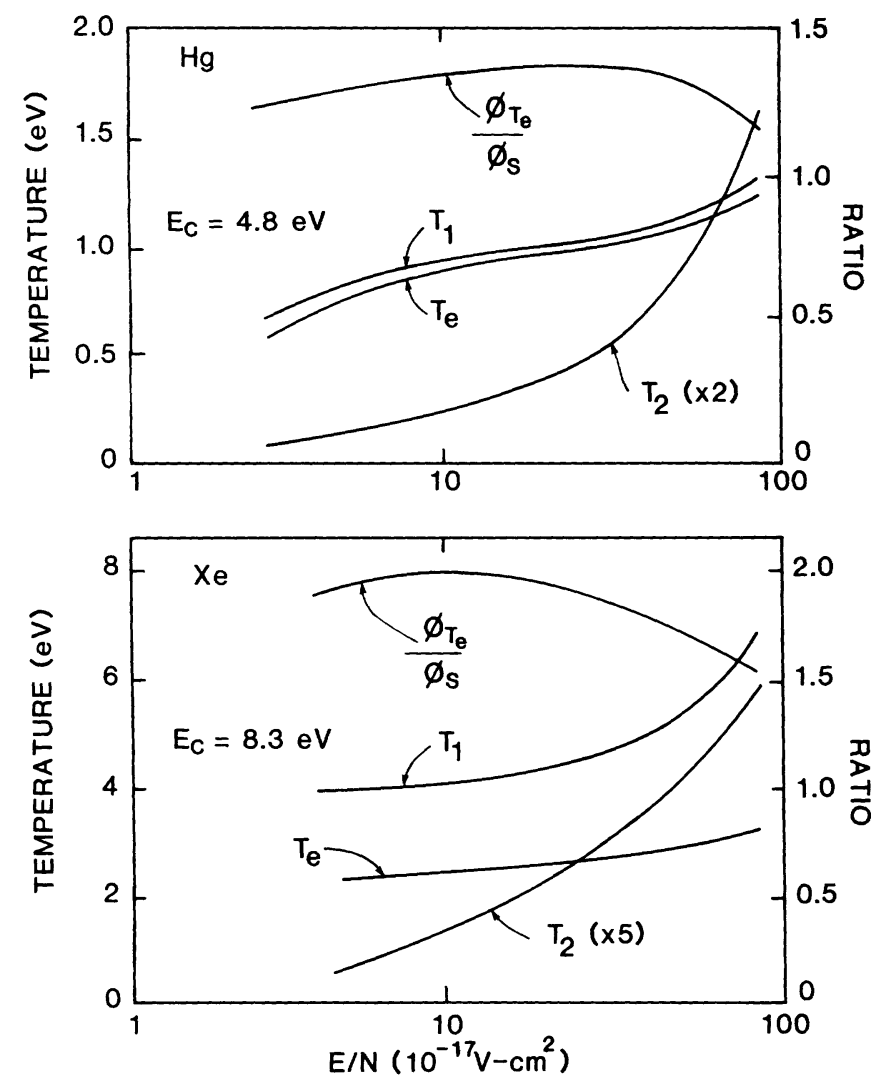

Fig. 3. Electron temperature $T_{e}, T_{1}$, and $T_{2}$ for discharges in $\mathrm{Hg}$ and $\mathrm{Xe}$ plotted as a function of $E / N$. These values were obtained from solution of Boltzmann's equation. Also plotted is $\phi_{T_{e}} / \phi_{s}$, the ratio of the sheath potential based on $T_{e}$ to that obtained using $T_{2} / T_{1}$ and $E_{c} / k T_{1}$. For sufficiently large $E / N, T_{2}$ approaches $T_{1}$ and $\phi_{T_{e}} / \phi_{s}$ approaches unity.

equation for the electron distribution function in the plasma. These solutions of Boltzmann's equation were obtained using the program described in [7]. $T_{e}, T_{1}$, and $T_{2}$ for discharges in $\mathrm{Hg}$ and $\mathrm{Xe}$ with applied fields of $3 \times 10^{-17}-10^{-15} \mathrm{~V} \cdot \mathrm{cm}^{2}$ are plotted in Fig. 3. (Other solutions to Boltzmann's equation for these discharge conditions can be found in [8] and [9].) The cutoff energies are 4.8 and $8.3 \mathrm{eV}$, respectively. For both gases, the cutoff energy is the threshold energy for excitation of the first electronic states. For these non-Maxwel- lian distributions, we define $\epsilon=3 k T_{e} / 2$, where $\epsilon$ is the average electron energy. Also plotted in Fig. 3 is $\phi_{T_{e}} / \phi_{s}$, the quovient of the sheath potential one would calculate based on $T_{e}$ divided by the sheath potential obtained using the parameters $T_{2} / T_{1}$ and $E_{c} / k T_{1}$. For a large range of $E / N, \phi_{T_{e}} / \phi_{s}$ remains fairly constant for both $\mathrm{Hg}$ and $\mathrm{Xe}$. As $E / N$ becomes sufficiently large, $T_{2}$ begins to approach $T_{1}$, and the distribution appears to be more Maxwellian. When this occurs the value of $\phi_{T_{e}} / \phi_{s}$ approaches unity.

In conclusion, the sheath potential of an isolated conductor or insulated surface immersed in a non-Maxwellian plasma was computed using a two-electron temperature model. The twotemperature model more accurately describes the depletion of electrons with energy greater than the first excitation threshold than does a Maxwellian distribution, and predicts a lower sheath potential than one would calculate with a Maxwellian. For typical glow discharges, the actual sheath potential is lower by many times the average electron energy than the value one would calculate using the bulk electron temperature.

\section{REFERENCES}

[1] M. Mitchner and C. H. Kruger, Jr., Partially Ionized Plasmas. New York: Wiley, 1973, pp. 129-134.

[2] W. L. Nighan, "Electron energy distributions and collision rates in electrically excited $\mathrm{N}_{2}, \mathrm{CO}$ and $\mathrm{CO}_{2}$," Phys. Rev. $A$, vol. 2, p. $1989,1970$.

[3] L. Vriens, "Energy balance in low pressure gas discharges," $J$. Appl. Phys., vol. 44, p. 3980, 1973.

[4] L. Vriens, "Two and three electron group models for low pressure gas discharges," J. Appl. Phys., vol. 45, p. 1191, 1974.

[5] L. Vriens and F. A. S. Ligthart, "Energy balance and coulomb relaxation in low pressure gas discharges," Philips Res. Rep., vol. 32, p. $1,1977$.

[6] W. L. Morgan and L. Vriens, "Two-electron-group model and Boltzmann calculations for low pressure gas discharges," J. Appl. Phys., vol. 51, p. 5300, 1980.

[7] W. L. Morgan, "Elendif': A computer program that solves the Boltzmann equation for a partially ionized gas," Joint Institute of Laboratory Astrophysics Information Center, Univ. of Colorado, Boulder, CO, Rep. 19, June 1979.

[8] O. Judd, "Electron-collisional excited-state kinetics in argon and mercury electrical discharges," J. Appl. Phys., vol. 47, p. 5297, 1976.

[9] M. Hayashi, "Calculation of swarm parameters in xenon at high $E / N$ by a Monte Carlo simulation method," J. Phys. D., vol. 16, p. 591, 1983. 